\title{
Quantification of Plant Dormancy
}

\author{
held at the \\ 92nd ASHS Annual Meeting \\ Montreal, Québec, Canada \\ 2 August 1995
}

sponsored by the
Plant Dormancy Research Working Group

published by the

American Society for Horticultural Science

Alexandria, VA 22314-2562

as a special insert in

HortScience 32(4), July 1997 


\title{
Workshop Papers and Authors
}

\author{
Presiding: Anwar A. Khan
}

Quantification of Plant Dormancy: Introduction to the Workshop

Anwar A. Khan

Quantification of Seed Dormancy: Physiological and Molecular Considerations

Anwar A. Khan

Quantification of Endodormancy in Seeds of Woody Plants

Schuyler D. Seeley

Quantification of Bud Dormancy: Physiological Approaches

Leslie H. Fuchigami and Michael Wisniewski

Bud Dormancy in Perennial Fruit Trees: Physiological Basis for Dormancy Induction, Maintenance, and Release

Miklos Faust, Amnon Erez, Lisa J. Rowland, Shiow Y. Wang, and H.A. Norman

\section{Quantification of Plant Dormancy: Introduction to the Workshop}

\author{
Anwar A. Khan \\ Department of Horticultural Sciences, New York State Agricultural Experiment Station, Cornell University, \\ Geneva, NY 14456
}

Dormancy is a physiological state that reduces the ability of a seed or bud to germinate or grow. Dormancy is controlled by 1) factors residing in the embryo or bud (endodormancy, primary dormancy); 2) plant-situated factors outside the embryo or bud, such as covering structures or a terminal bud, which may exert a mechanical, chemical, or correlative inhibition (paradormancy, coat-imposed dormancy); and 3) environmental factors, such as high temperature, unfavorable irradiation or photoperiod, a low water potential sowing medium, applied chemical inhibitors, etc. (ecodormancy, induced dormancy). Although a variety of terms have been used to describe dormancy types in seeds and buds, based on the factors that influence dormancy, the underlying mechanism controlling embryo and bud dormancy may be the same (Khan, 1975). Stress factors, such as noted under 2) and 3) above, can induce dormancy by affecting the dormancy regulating mechanism, but their effect appears to be indirect. Factors such as farred irradiation and gibberellin (GA) synthesis inhibitors appear to induce dormancy more rapidly, at least in seeds, indicating that their action is more direct (Khan, 1994). Similarly, GA, irradiation and moist-chilling are directly involved in dormancy release and prevention, unlike the stress-alleviating factors, such as cytokinins and ethylene, which indirectly affect dormancy release.

Measurable changes in germination or growth potential in the embryo or bud occurring naturally or induced by artificial means can be used to quantify dormancy. These include changes in the mechanical constraints of the covering structures (seedcoat, bud scales), water potential of the medium, hormone levels, synthesis of macromolecules, duration of exposure to chilling temperatures or chilling units, duration of soaking in darkness (for seeds with skotodormancy), the amount of indoleacetic acid (IAA) replacing the terminal bud, etc., in relation to dormancy induction, intensification, or release. The quantification of dormancy using this approach is largely correlative and

Received for publication 1 July 1996. Accepted for publication 29 Nov. 1996. The cost of publishing this paper was defrayed in part by the payment of page charges. Under postal regulations, this paper therefore must be hereby marked advertisement solely to indicate this fact. does not deal directly with the inherent causes of embryo or bud dormancy.

Dormancy induction and release in seeds has been shown to be a reversible process, occurring under a wide variety of conditions (Khan, 1994). The evidence to date points to an association of dormancy release and maintenance with phase transitions and compositional changes in cell membranes. Dormancy release is activated by membrane-active agents, including red light, brief high-temperature shifts, osmotic shock, anaesthetics, and moist-chilling. These agents initiate production of an array of bioactive chemicals, some of which (e.g., GAs) may act more directly than others to release the dormancy. Dormancy release or prevention by GA or irradiation is independent of germination and can be shown under conditions when germination is inhibited, e.g., by high temperature, low water potential, or abscisic acid (ABA). The inability to produce active GAs may be related to dormancy maintenance.

Studies with seeds and buds indicate that dormancy release by GA, irradiation, decapitation, or moist-chilling is associated with enhanced expression of certain genes; in the absence of these treatments, genes are not expressed (Stafstrom and Sussex, 1993; Toyomasu et al., 1995). In other cases, the transcript levels of other genes increases in dormant embryos and nondormant embryos exposed to ABA or a stressful environment, while the levels decline rapidly in nondormant embryos (Goldmark et al., 1992; Li and Foley, 1995). Thus, a major difficulty has been to distinguish between stress-related (imposition or alleviation) gene expression from that associated with dormancy induction and release. In absence of a clear understanding of the underlying mechanism of bud and embryo dormancy, conflicting theories are bound to appear.

The papers presented at this workshop discuss the various factors influencing dormancy in seeds and buds and the conceptual framework necessary for quantifying dormancy. The first two articles are devoted to dormancy quantification in seeds and the next two to the quantification in buds. The article by Khan describes the various physiological approaches that have been used to quantify dormancy and touches upon the molecular approaches indicative of dormancy 